\title{
Towards a Rigorous Proof of Magnetism on the Edges of Graphene Nano-ribbons
}

\author{
Hamed Karimi and Ian Affleck \\ Department of Physics and Astronomy, University of British Columbia, Vancouver, B.C., Canada, V6T 1Z1
}

(Dated: July 31, 2021)

\begin{abstract}
A zigzag edge of a graphene nanoribbon supports localized zero modes, ignoring interactions. Based mainly on mean field arguments and numerical approaches, it has been suggested that interactions can produce a large magnetic moment on the edges. By considering the Hubbard model in the weak coupling limit, $U \ll t$, for bearded as well as zigzag edges, we argue for such a magnetic state, based on Lieb's theorem. Projecting the Hubbard interactions onto the flat edge band, we then prove that resulting 1 dimensional model has a fully polarized ferromagnetic ground state. We also study excitons and the effects of second neighbor hopping as well as a potential energy term acting on the edge only, proposing a simple and possibly exact phase diagram with the magnetic moment varying smoothly to zero. Finally, we consider corrections of second order in $U$, arising from integrating out the gapless bulk Dirac excitations.
\end{abstract}

One of the many fascinating properties predicted for graphene 1 is that a non-interacting nanoribbon with zigzag edges has bands of states with energy exponentially small in the ribbon width, localized at the edges 2. Unzipping of carbon nanotubes has recently provided a technique for producing nano-ribbons with clean edges and Scanning Tunnelling Microscopy (STM) on such ribbons [3] has shown evidence for interacting edge states. It has been proposed, on the basis of mean field theory [2, 4, 5], density functional theory [6, 7] and various numerical techniques [8 10], that Hubbard interactions may induce ferromagnetic order of the electrons in these edge states, with the moments on opposite edges ordering antiferromagnetically. Graphene edge magnetism looks promising for applications in nanoelectronics [11. However, there is no experimental evidence for edge magnetism in graphene ribbons and it is a matter of debate whether it is sufficiently robust to occur in realistic models [12, thus motivating a deeper and more general understanding of its origins. One might try to regard this magnetism as an essentially one dimensional (1D) phenomenon, since it arises from edge states, but the 1D Hubbard model is known to have a non-ferromagnetic ground state at all doping, being antiferromagnetic at half-filling. Recently [13, 14, the effective 1D model obtained by projecting the Hubbard interactions onto the edge states was studied numerically, and argued to lead to ferromagnetic order of an isolated edge. An interesting limit in which to try to prove edge magnetism is the weak interaction limit of the Hubbard model, $U \ll t$, at half-filling. We take two steps towards proving edge magnetism in this limit. The first involves applying Lieb's theorem [15] to the contrasting cases of a nanoribbon with two zigzag edges (ZZ) versus a ribbon with one zigzag and one bearded edge (ZB). (See Figure 1.) Then we prove that the projected 1D Hamiltonian has a fully polarized ferromagnetic ground state. We also study numerically other properties of the 1D model, obtaining the electron or hole addition energy and showing that there are bound spin-1 excitons. We then consider two important particle-hole symmetry breaking perturbations: second neighbor hopping, $t_{2}$ (in the entire ribbon) and a potential energy, $V_{e}$, acting on the edge atoms only, with the chemical potential maintained at the Dirac points of the bulk dispersion relation. Both perturbations lead 17 to the same new term in the 1D Hamiltonian, $\propto t_{2}-V_{e} \equiv \Delta$. We argue that the fully polarized ground state survives up to a critical value of $|\Delta|$ of $O(U)$, beyond which the ground state may still be found exactly and has a smoothly decreasing edge magnetic moment. Integrating out the bulk excitations of the ribbon leads to both inter-edge and intra-edge interactions. The inter-edge interactions $\propto U^{2} /\left(t W^{2}\right)$, where $W$ is the ribbon width, produce antiferromagnetic order for the ZZ ribbon but ferromagnetic order for the ZB ribbon. Intra-edge interactions, $\propto U^{2} / t$, exhibit only a mild logarithmic singularity at low energies, arising from the gapless nature of the bulk Dirac excitations.

We consider the Hubbard model at half-filling on a long ribbon of honeycomb lattice with periodic boundary conditions in the $x$-direction and zigzag edges. We actually find it convenient to first consider an upper zigzag edge and a lower bearded edge. [See Figure 1.] Let the number of atoms along the zigzag edge, of $A$ type, be $L$. (Therefore the length of the ribbon is $\sqrt{3} a L$ where $a$ is the nearest neighbour separation. We generally set $\sqrt{3} a=1$.) $L$ is also the number of hairs in the beard, ending at $A$ sites. Noting that the number of $A$ sites minus the number of $B$ sites is $L$, it follows from Lieb's theorem [15], that the ground states have spin $S=L / 2$ for all values of $U / t>0$. We label the width of the strip by another positive integer, $W$ so that the width is $3 a W / 2$ in the ZB case or $a(3 W+1) / 2$ in the ZZ case. For the non-interacting model, $U=0$, and $W, L \gg 1$, there are approximately $L / 3$ zero energy states localized at the upper zigzag edge and $2 L / 3$ localized at the lower bearded edge. The zigzag edge states have wave-vectors, $2 \pi / 3 \leq|k|<\pi$ while the bearded edge states fill the rest of the Brillouin zone, $|k| \leq 2 \pi / 3$. It follows from particle-hole symmetry that all of these states have exactly zero energy (for any $W$ ). These $L$ zero modes are half-filled so we have a large degeneracy of ground states including a spin multiplet with spin $L / 2$ obtained by filling 


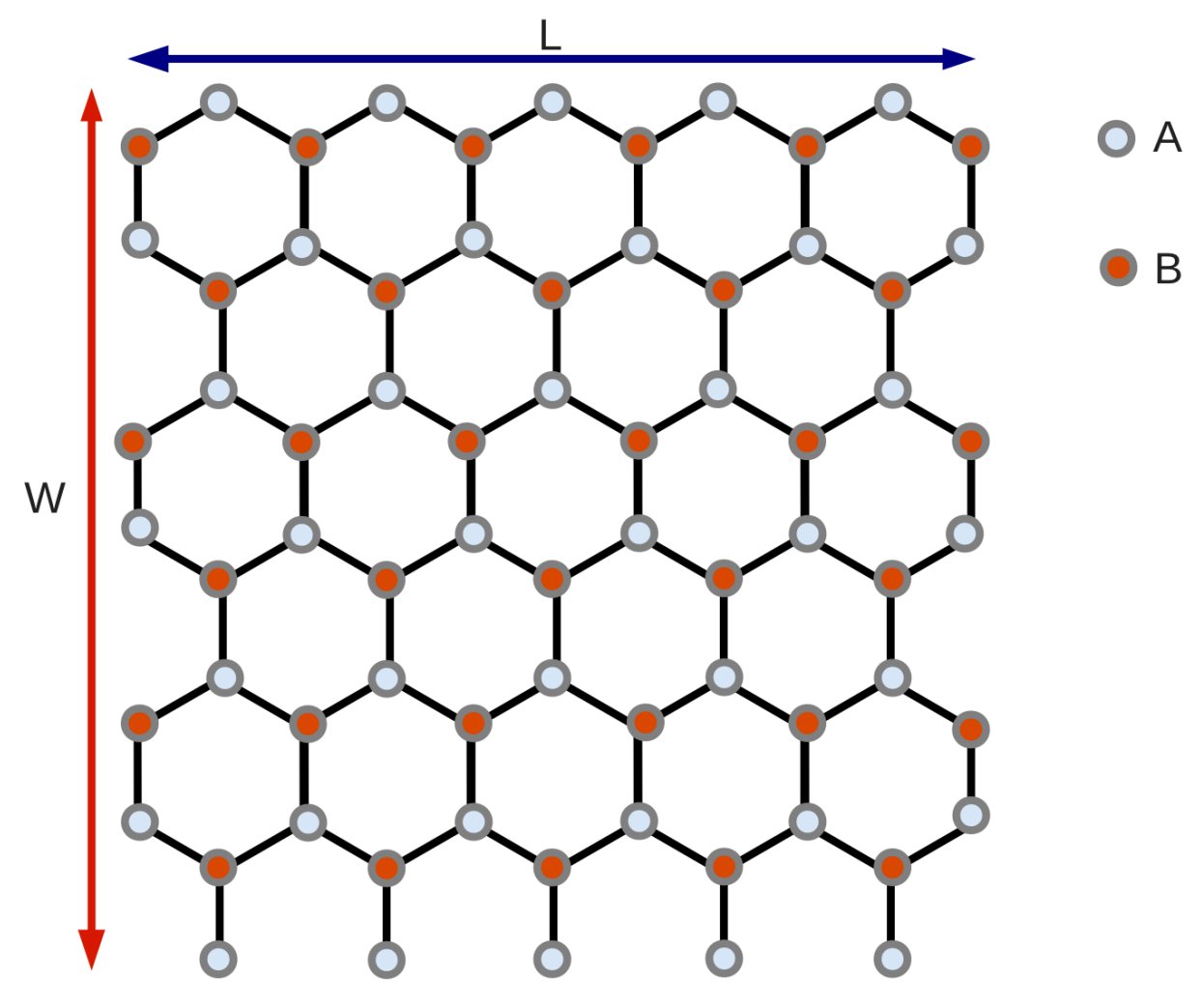

FIG. 1. A nanoribbon with an upper zigzag edge and lower bearded edge. In this example, $L=5$ and $W=6$. (We employ periodic boundary conditions in the $x$-direction.)

each of the $L$ states with a single electron with the same polarization. Now consider turning on a very small positive $U$, in the case of large $W$ and $L$. In the absence of boundaries we don't expect $U$ to have a large effect on the ground state. A gapless non-magnetic Dirac liquid state is expected to persist up to a critical $U_{c}$ of $O(t)$. This follows from the fact that perturbation theory in $U$ is infrared finite in the Dirac model; i.e. the 4-fermi interaction is irrelevant. Therefore, we expect the magnetic moment to live on the edges. The only physical explanation of the Lieb's theorem result seems to be that the fully polarized edge state multiplet persists as the (unique) ground state as we turn on $U$. This picture can be further substantiated by calculating the weak interaction between the upper and lower edges, of order $U^{2} /\left(t W^{2}\right)$. This interaction is found to be ferromagnetic, as we show below. Thus there must be a spin $\approx L / 6$ on the zigzag edge and $\approx L / 3$ on the bearded edge, with these two spins coupled ferromagnetically. Now consider replacing the bearded edge by a second zigzag edge. Lieb's theorem [15] now implies a zero spin ground state since we have equal numbers of $A$ and $B$ sites. Now, at $U=0$, we have approximately $L / 3$ edge states on both lower and upper edges. These mix to form two bands, with $2 \pi / 3 \leq k \leq 4 \pi / 3$, with energies exponentially small in $W$ and symmetric around $E=0$. Ignoring inter-edge interactions, we expect spin $\approx L / 6$ on both upper and lower edges. In this case, the intra-edge interaction of order $U^{2} /\left(t W^{2}\right)$ is antiferromagnetic, implying a zero spin ground state consistent with the result from Lieb's theorem. A further consistency check can be obtained by going smoothly between zigzag and bearded lower edges by turning on the hopping term, $t^{\prime}$ on the hairs. Lieb's theorem implies spin $L / 2$ for all $t, t^{\prime}$ and $U>0$. For $t^{\prime}=0$ we have a ZZ ribbon together with $L$ decoupled sites sitting below the lower edge. The ZZ ribbon has spin 0 but we can obtain a state with total spin $L / 2$ by polarizing the electron spins at the decoupled sites. Although the zigzag ribbon has total spin 0 , for large $W$ we expect that the upper and lower edges have spin $\approx L / 6$ with antiferromagnetic inter-edge coupling. Turning on $t^{\prime}$ produces an effectively antiferromagnetic coupling between the spin $\approx L / 6$ on the lower zigzag edge and $L / 2$ on the nearly decoupled sites. This gives a moment $\approx L / 3$ which is now ferromagnetically coupled to the upper edge, giving a total spin of $L / 2$ as required by Lieb's theorem.

Assuming that the ground state remains an unpolarized Dirac liquid up to $U=U_{c}$, the magnetism of the edges 
seems to follow from Lieb's theorem for large $W$. If the transition at $U_{c}$ is into a bulk antiferromagnetic state (with, for example, spin up on $A$ sites and spin down on $B$ sites) then the edge magnetism should persist, since it is of this type, and may be regarded as a sort of precursor of the bulk antiferromagnetic order. The simplest possibility is that the system goes from Dirac liquid into Mott-Hubbard insulator at $U_{c}$ but numerical evidence [18] has been presented for a spin liquid phase at intermediate $U$, of unknown edge magnetic properties.

We note that the above arguments also apply to carbon nanotubes [19. Indeed, since we have been considering periodic boundary conditions in the $x$-direction, we have actually been discussing tubes, of circumference $L$ and length $W$. The magnetic moments exist on the upper and lower caps (i.e. rings) of the nanotubes with ferromagnetic or antiferromagnetic inter-ring coupling for a bearded or zigzag lower ring respectively. (The half-filled bulk of the nanotube might be in a 1D version of a Mott-Hubbard insulating state but this only serves to weaken the effects of bulk states on edge states.)

We may further substantiate this picture by considering [13] the weak intra-edge interactions of $O(U)$. Simply projecting the Hubbard interaction onto the zero energy states on the zigzag edge, in the large $W$ limit, gives a Hamiltonian:

$$
\mathcal{H}=\frac{1}{2} \sum_{k, k^{\prime}, q} \Gamma\left(k, k^{\prime}, q\right)\left[\sum_{\sigma} e_{\sigma}^{\dagger}(k+q) e_{\sigma}(k)-\delta_{q, 0}\right]\left[\sum_{\sigma^{\prime}} e_{\sigma^{\prime}}^{\dagger}\left(k^{\prime}-q\right) e_{\sigma^{\prime}}\left(k^{\prime}\right)-\delta_{q, 0}\right]+E_{0}
$$

Here $e_{\alpha}(k)$ annihilates an electron in an edge state with momentum $k$ and spin $\alpha=\uparrow$ or $\downarrow$. The interaction function is:

$$
\Gamma\left(k, k^{\prime}, q\right)=\frac{\left\{\left[1-\left(2 \cos \frac{k}{2}\right)^{2}\right]\left[1-\left(2 \cos \frac{k+q}{2}\right)^{2}\right]\left[1-\left(2 \cos \frac{k^{\prime}}{2}\right)^{2}\right]\left[1-\left(2 \cos \frac{k^{\prime}-q}{2}\right)^{2}\right]\right\}^{1 / 2}}{1-16 \cos \frac{k}{2} \cos \frac{k+q}{2} \cos \frac{k^{\prime}-q}{2} \cos \frac{k^{\prime}}{2}}
$$

The sum over $k, k^{\prime}$ and $q$ is restricted to the band in which $2 \pi / 3<k, k^{\prime}, k+q, k^{\prime}-q<4 \pi / 3$ and $\Gamma\left(k, k^{\prime}, q\right)$ is strictly positive [16. Periodic boundary conditions in the $x$-direction imply $k=2 \pi n / L$ so the number of wave-vectors $N \approx L / 3$. Note that we are considering the case of half-filling in the entire lattice and that the edge Hamiltonian is therefore invariant under the particle-hole symmetry transformation:

$$
e_{\alpha}(k) \leftrightarrow e_{\alpha}^{\dagger}(k)
$$

This is highly unusual since normally a particle-hole symmetry transformation relates a particle and hole at different wave-vectors. Here with an exactly flat band, the particle and hole operators occur at the same wave-vectors. It is important to note that $\Gamma\left(k, k^{\prime}, q\right)$ arose from summing the wave-function of the edge states over sites at arbitrary distance $n$ from the zigzag edge and can be written:

$$
\Gamma(l, k, q)=\sum_{n=0}^{\infty} g_{n}(k) g_{n}(l) g_{n}(l+q) g_{n}(k-q)
$$

where

$$
g_{n}(k) \equiv \theta\left(1-\left|2 \cos \frac{k}{2}\right|\right) \sqrt{1-\left(2 \cos \frac{k}{2}\right)^{2}}\left(2 \cos \frac{k}{2}\right)^{n}
$$

Thus, dropping the constant $E_{0}$, we may write:

$$
\mathcal{H}=\frac{1}{2} \sum_{n, q} O_{n}^{\dagger}(q) O_{n}(q)
$$

with

$$
O_{n}(q) \equiv \sum_{k} g_{n}(k) g_{n}(k+q)\left[\sum_{\sigma} e_{\sigma}^{\dagger}(k+q) e_{\sigma}(k)-\delta_{q, 0}\right]
$$

It follows that all eigenstates of $H$ are non-negative. It is can be seen that fully spin-polarized state is a zero energy eigenstate and therefore a ground state. To check this consider, for example, the representative fully polarized state 
where all electron spins are in the up direction. Then clearly $O_{n}(q)$ annihilates this state for all non-zero $q$ since the spin up terms in $O_{n}(q)$ try to produce a spin up electron in an occupied state with wave-vector $k+q$ while the spin down terms try to annihilate a spin down electron in a vacant state of wave-vector $k$. $O_{n}(0)$ also annihilates this state since the occupancy of each single particle state is precisely 1 .

It is also possible, though more difficult, to argue that the fully polarized multiplet, of spin $S=L / 6$, are the unique groundstates of the projected 1D Hamiltonian. To prove that fully polarized states are the unique ground states of $H$ we need to prove that the only states annihilated by $O_{n}(q)^{\dagger} O_{n}(q)$ for all $n$ and $q$ are fully polarized (that is, have maximal total spin). For convenience, in this paragraph, we take all momenta to be in the region of $[-\pi / 3, \pi / 3]$, which can be obtained by shifting all of them by $\pi$. Suppose $|\psi\rangle$ is such that for any $n, q, O_{n}(q)|\psi\rangle=0$. Then we have

$$
\begin{aligned}
& \sum_{k} g_{n}(k) g_{n}(k+q)\left[e_{\sigma}^{\dagger}(k+q) e_{\sigma}(k)-\delta_{q, 0}\right]|\psi\rangle=0 \\
= & \sum_{k>0} g_{n}(k) g_{n}(k+q)\left[e_{\sigma}^{\dagger}(k+q) e_{\sigma}(k)+e_{\sigma}^{\dagger}(-k) e_{\sigma}(-k-q)-2 \delta_{q, 0}\right]|\psi\rangle=0
\end{aligned}
$$

since $g_{n}(k) g_{n}(k+q)=g_{n}(-k) g_{n}(-k-q)$. (Repeated spin indices are summed in this section.) For fixed $q$ and using the definition of $g_{n}$ we have, for any $n$ that

$$
\begin{gathered}
\sum_{k>0}\left(4 \sin \left(\frac{k}{2}\right) \sin \left(\frac{k+q}{2}\right)\right)^{n}\left|\psi_{k}^{(q)}\right\rangle=0 \\
\left|\psi_{k}^{(q)}\right\rangle \equiv \sqrt{1-(2 \sin k / 2)^{2}} \sqrt{1-(2 \sin (k+q) / 2)^{2}}\left[e_{\sigma}^{\dagger}(k+q) e_{\sigma}(k)+e_{\sigma}^{\dagger}(-k) e_{\sigma}(-k-q)-2 \delta_{q, 0}\right]|\psi\rangle
\end{gathered}
$$

Since $n$ runs from 0 to $\infty$, the number of independent momenta is $L / 3$ and all the $\left(4 \sin \left(\frac{k}{2}\right) \sin \left(\frac{k+q}{2}\right)\right)$ are different, the determinant of the Vandermonde matrix is non-zero so Eq. (9) is satisfied if and only if for any $k, q$ we have

$$
\left[e_{\sigma}^{\dagger}(k+q) e_{\sigma}(k)+e_{\sigma}^{\dagger}(-k) e_{\sigma}(-k-q)-2 \delta_{q, 0}\right]|\psi\rangle=0
$$

First, using Eq. (11) for $q=0$, we get $n(k)+n(-k)=2$, thus the only possible terms have $n(k)=n(-k)=1$ or $n(k)=0$ and $n(-k)=2$ or vice versa. In general $|\psi\rangle$ could be written as a linear combination of Fock states $\prod c_{\sigma}^{\dagger}(k) \mid 0>$. We first will show that in the expansion of $|\psi\rangle$ in terms of such states there is no Fock state which for any momentum $k$ we have a vacancy or double occupancy in that momentum state. In other words, in the expansion of $|\psi\rangle$, with condition (11) and $n(k)+n(-k)=2$, only Fock states with singly occupied momentum states are allowed. Suppose that there is a state which has the property $n(k)+n(-k)=2$ for any $k$ and has double occupancy at momentum $l$ (vacancy at momentum $-l$ ); call this state $\phi$ :

$$
|\phi\rangle=|\cdots, \underbrace{0}_{-l}, \cdots, \underbrace{\downarrow \uparrow}_{l}, \cdots\rangle
$$

and we suppose $\langle\phi \mid \psi\rangle \neq 0$. We impose the condition 11 on $|\psi\rangle$ for $q=-2 l$ and $k=l$. Thus we should have $e_{\sigma}^{\dagger}(-l) e_{\sigma}(l)|\psi\rangle=0$. Let us first look at the action of $e_{\sigma}^{\dagger}(-l) e_{\sigma}(l)$ on $\phi$,

$$
\begin{aligned}
& e_{\sigma}^{\dagger}(-l) e_{\sigma}(l)|\phi\rangle=e_{\sigma}^{\dagger}(-l) e_{\sigma}(l)|\cdots, \underbrace{0}_{-l}, \cdots, \underbrace{\downarrow \uparrow}_{l}, \cdots\rangle \\
= & |\cdots, \underbrace{\uparrow}_{-l}, \cdots, \underbrace{\downarrow}_{l}, \cdots\rangle-|\cdots, \underbrace{\downarrow}_{-l}, \cdots, \underbrace{\uparrow}_{l}, \cdots\rangle
\end{aligned}
$$

Now, in order to satisfy the condition $e_{\sigma}^{\dagger}(-l) e_{\sigma}(l)|\psi\rangle=0$, we should have some other Fock states in the expansion of $|\psi\rangle$ such that the action of $e_{\sigma}^{\dagger}(-l) e_{\sigma}(l)$ on them could cancel the terms created in the second line of Eq. (13). $e_{\sigma}^{\dagger}(-l) e_{\sigma}(l)$ only acts on states with momentum $l,-l$ thus does not change the spin configurations of the other (singly occupied) states. There are only three possible Fock states which have the same configurations of the singly occupied states:

$$
|1\rangle=|\cdots, \underbrace{\downarrow \uparrow}_{-l}, \cdots, \underbrace{0}_{l}, \cdots\rangle \quad e_{\sigma}^{\dagger}(-l) e_{\sigma}(l)|1\rangle=0
$$




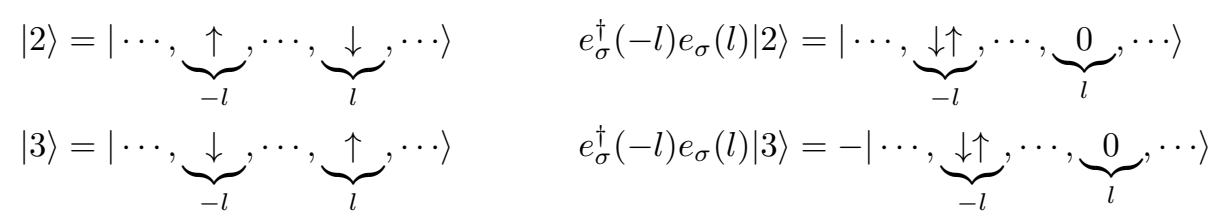

We see that none of these states are able to cancel the terms created on $(13)$. Then the assumption is wrong and we have to have $\langle\phi \mid \psi\rangle=0$. Having proven this, we show that in the expansion of $|\psi\rangle$ in terms of singly occupied Fock states, only symmetric combinations like the following are acceptable

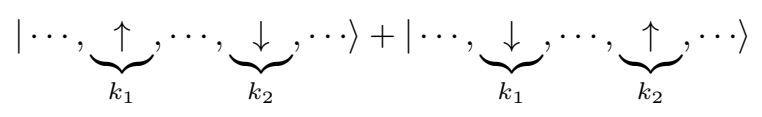

Suppose that, the Fock expansion of $|\psi\rangle$ has a term like $|\cdots, \underbrace{\uparrow}_{k_{1}}, \cdots, \underbrace{\downarrow}_{k_{2}}, \cdots\rangle$. Now by chosing $k=k_{1}$ and $q=k_{2}-k_{1}$ we should have $\left(e_{\sigma}^{\dagger}\left(k_{2}\right) e_{\sigma}\left(k_{1}\right)+e_{\sigma}^{\dagger}\left(-k_{1}\right) e_{\sigma}\left(-k_{2}\right)|\psi\rangle=0\right.$. We also have

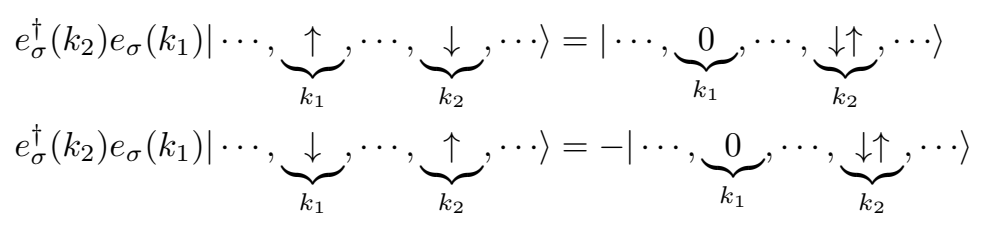

Thus we see that the symmetric combinations leads to zero, while the anti-symmetric ones give us a non-zero result. Thus $|\psi\rangle$ must be fully symmetric under exchanging all spins and is therefore of maximal spin.

One interesting quantity that follows from the Hamiltonian is the energy to add a spin down electron of momentum $k$, which is the same as the energy to remove a spin up electron of momentum $k$ :

$$
\epsilon_{k}=\frac{U}{2 L} \sum_{k^{\prime}} \Gamma\left(k, k^{\prime}, 0\right)
$$

This quantity is plotted (at $L \rightarrow \infty$ ) in Fig. 2. It vanishes linearly at the Dirac points, $k=2 \pi / 3,4 \pi / 3$. In principle, $\epsilon_{k}$ could be measured in Angular Resolved Photo-emission Spectroscopy (ARPES) experiments. The corresponding electron addition or removal energy is given by $\epsilon_{k}$. The corresponding density of states $\propto 1 /\left|d \epsilon_{k} / d k\right|$ could be measured by Scanning Tunnelling Microscopy (STM). With a spin-polarized STM tip and an edge fully polarized in the $z$-direction, it would only be possible to tunnel in a spin-down electron or tunnel out a spin-up electron.

We have calculated numerically the lowest energy particle-hole state of total momentum $q$, for $L$ up to $602(N=200)$. This is plotted in Fig. 3 along with the bottom of the particle-hole continuum. We see that a strongly bound exciton exists for most values of $q$, as might be expected in this strongly interacting system. However, the binding energy vanishing at $q= \pm 2 \pi / 3$. This vanishing can be understood from the fact that $\Gamma\left(k, k^{\prime}, q\right)$ vanishes when $k$ or $k^{\prime}$ is at a band edge $2 \pi / 3$ or $4 \pi / 3$ so the zero energy particle and hole become non-interacting at wave-vector $2 \pi / 3$ and $-2 \pi / 3$ or vice versa.

While Lieb's theorem continues to imply a fully polarized ground state at sufficiently small $U$ for any hopping terms between opposite sub-lattices $(A$ to $B)$, adding a small $[\mathrm{O}(U)]$ second neighbor hopping term, $t_{2}$, may destroy the fully polarized state. Likewise, a single site potential, $V_{e}$, acting at the edge of the ribbon only, could destroy the fully polarized state. Temporarily ignoring interactions, the zigzag edge states survive at finite $t_{2}$ and $V$ but develop a non-zero dispersion given, to first order in $\Delta \equiv t_{2}-V_{e}$, by [17]: $\epsilon_{2}(k)-\epsilon_{F}=\Delta(2 \cos k+1)$. breaking the particle-hole symmetry. Here we are assuming, for simplicity, that the bulk chemical potential is at the energy of the bulk Dirac points, which becomes $\epsilon_{F}=3 t_{2}$. (Shifting $\epsilon_{F}$ away from the Dirac points, the Hubbard interactions have a larger effect in the bulk rendering the edge model approach more questionable.) Including a small $U$, the energy to add a spin down electron or remove a spin up electron at momentum $k$ now becomes

$$
E_{p / h}(k)=\epsilon_{k} \pm \Delta(2 \cos k+1) .
$$

respectively, where $\epsilon_{k}$ is given in Eq. (17). $E_{p}(k)$ is plotted in Fig. 2 for several values of $\Delta$. We see that for $|\Delta|<\Delta_{c} \approx 0.109 U$, the energy to add an electron or hole remains positive, so the edge states remain undoped. A local minimum at $k=\pi$ develops in $E_{p}(k)$ for $\Delta>.087 U$, and $E_{p}(k)$ becomes negative in the vicinity of $k=\pi$ for $\Delta>\Delta_{c}$. The lowest energy of a particle-hole state, and the bottom of the particle-hole continuum for various 


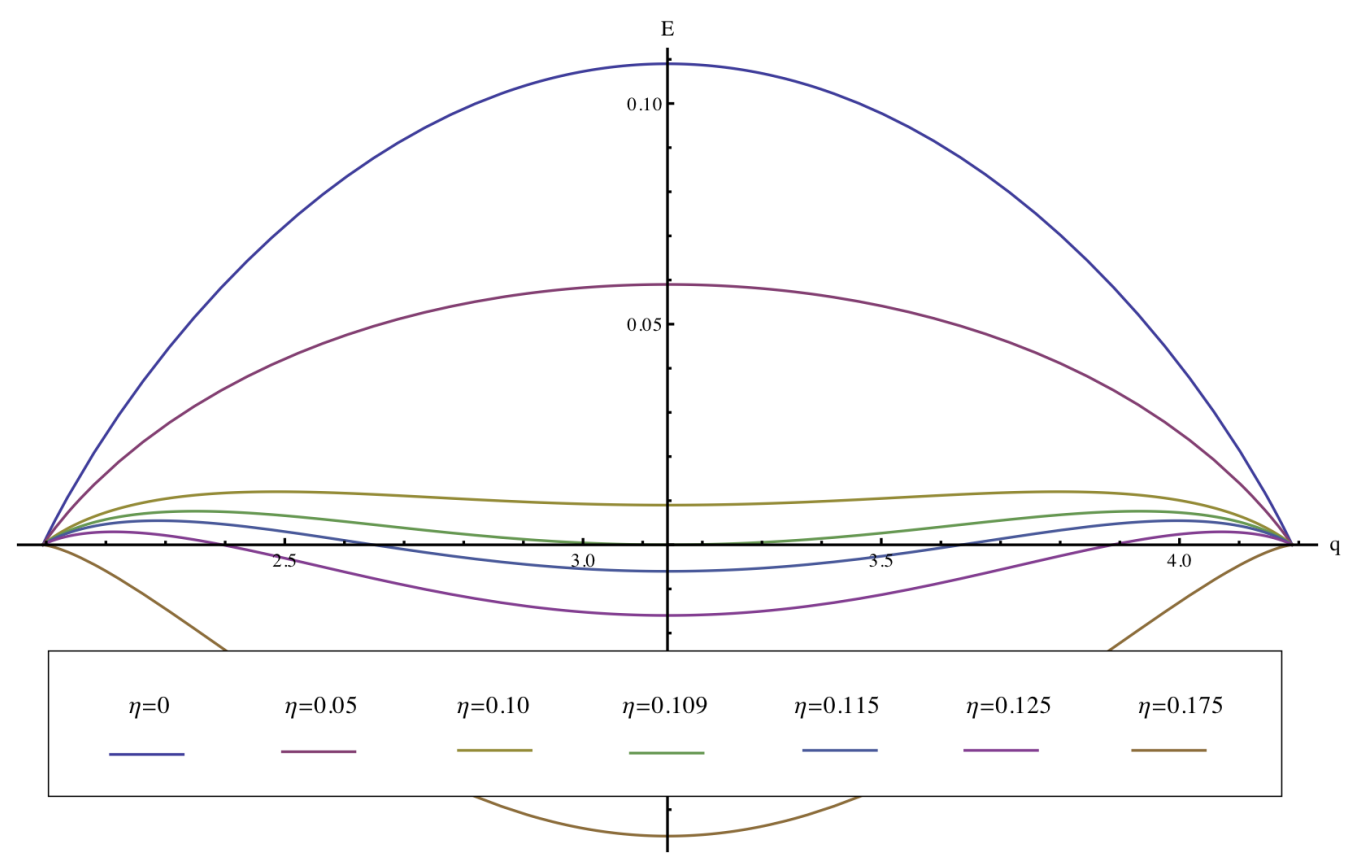

FIG. 2. Energy to add a spin down electron of momentum $k$ for various values of $\eta \equiv \Delta / U \equiv\left(t_{2}-V_{e}\right) / U$.

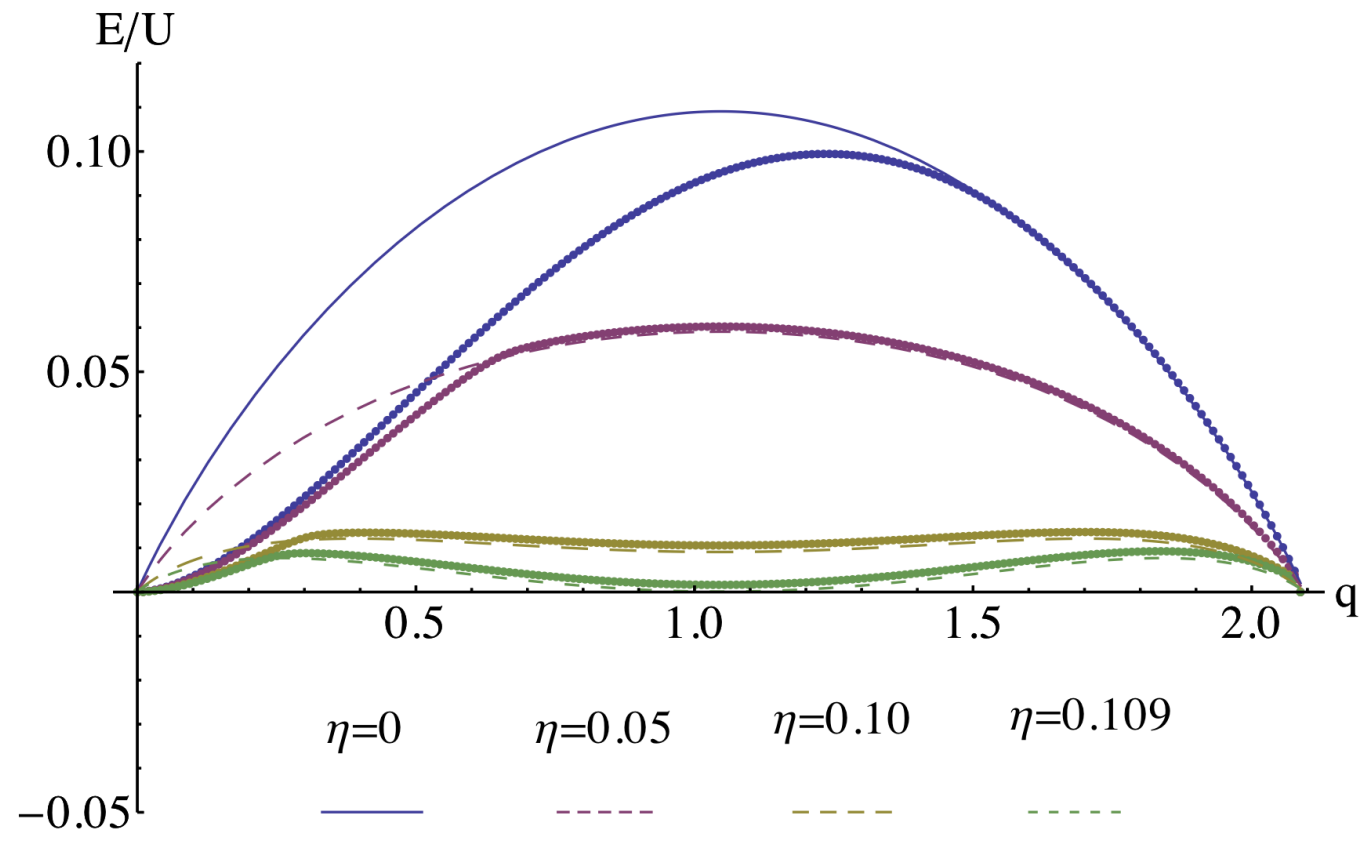

FIG. 3. Lowest energy particle-hole state (circles) and bottom of the particle-hole continuum (lines) for various values of $\eta \equiv \Delta / U \equiv\left(t_{2}-V_{e}\right) / U$. 


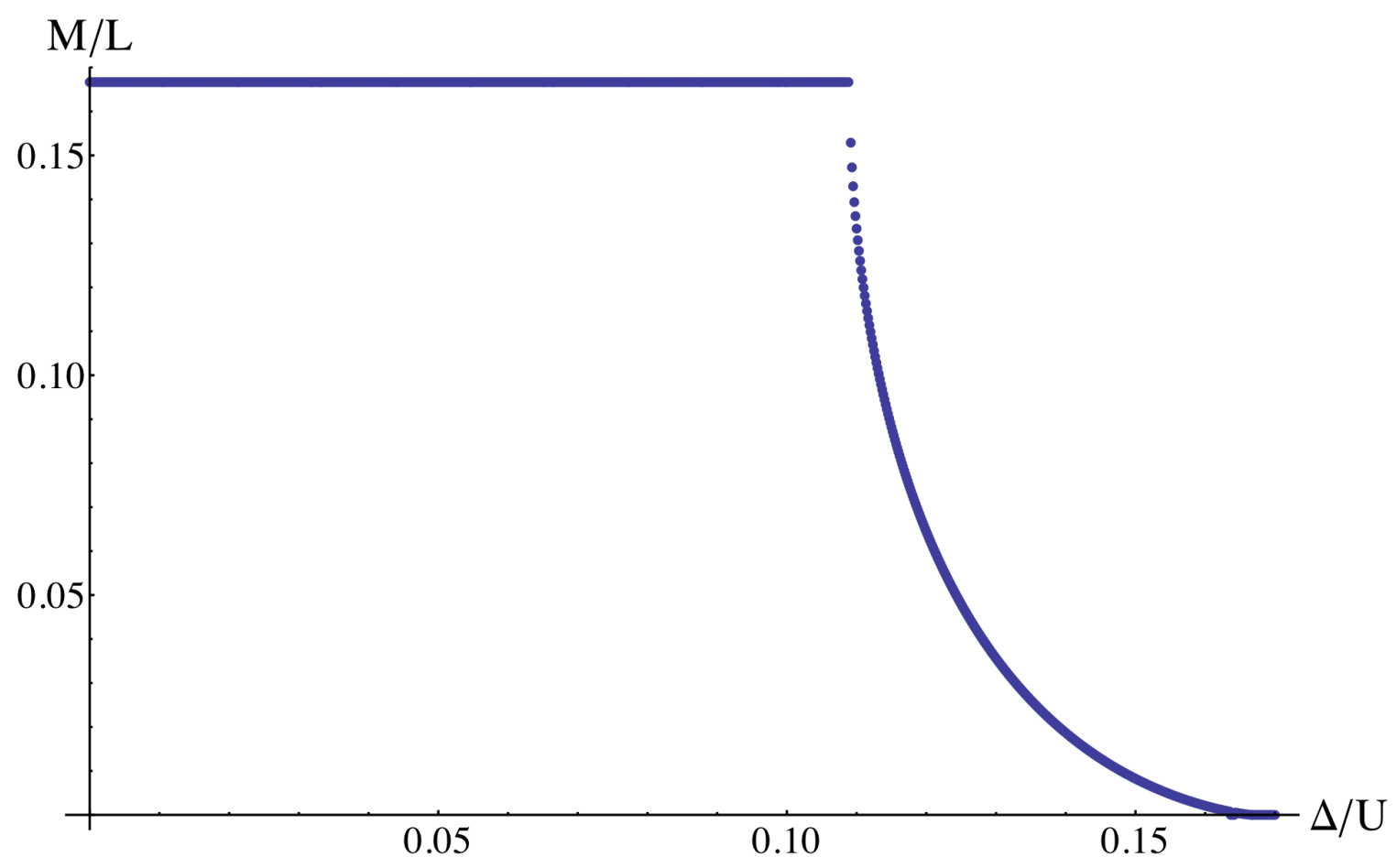

FIG. 4. Edge magnetization versus $\Delta \equiv t_{2}-V_{e}$.

values of $\Delta$ are shown in Fig. 3 . We see that the exciton becomes unbound except for wave-vectors near zero, as $|\Delta|$ increases. For $|\Delta|>\Delta_{c}$ the edge states become doped, adsorbing electrons or holes from the bulk. The simplest assumption for $|\Delta|>\Delta_{c}$ is that a Fermi sea of spin-down electrons or spin up holes forms near $k=\pi$, for $\Delta>\Delta_{c}$ or $\Delta<-\Delta_{c}$ respectively. This assumption is reasonable since there appear to be no bound excitons for $\Delta>\Delta_{c}$. We also calculated for $\Delta$ near $\Delta_{c}$ and $L \leq 74$, the lowest energy state with $M=N / 2-2$, finding no states below the 2-particle, 2-hole continuum, consistent with this assumption. These are exact eigenstates of the Hamiltonian of Eq. (1), 18 with the added holes or particles non-interacting. The corresponding exact result for magnetization versus $\Delta$ is plotted in Fig. 4, given this assumption. The non-interacting nature is a simple consequence of the fact that the on-site Hubbard model only gives interaction between electrons of opposite spin. On the other hand, we cannot rule out the possibility that the ground state for $\Delta>\Delta_{c}$ contains a finite density of spin-up holes as well as the spin-down electrons (and similarly for $\Delta<-\Delta_{c}$ ). In that case, Hubbard interactions have a non-trivial effect. In any event, adding a nearest neighbour Coulomb repulsion term to the bulk Hamiltonian has no effect on the projected edge Hamiltonian, since such a term acts between $A$ and $B$ sites whereas the zigzag edge states live entirely on one sublattice. On the other hand, a second neighbour Coulomb repulsion, $U_{2}$, produces interactions between electrons with parallel spins in the projected edge Hamiltonian. While this doesn't change our conclusions qualitatively in the undoped phase, it will produce interaction effects in the ground state for the doped case even if contains only particles or only holes. However, we might expect $U_{2} \ll U$, in which case these effects could be quite small. Thus in general we expect a one or two component Luttinger liquid for $|\Delta|>\Delta_{c}$. On the other hand the edge phase occurring for $|\Delta|<\Delta_{c}$ is definitely not a Luttinger liquid. Instead, it might be described as a fully spin-polarized semi-metal since all levels are filled with spin-up electrons and there is a non-zero electron and hole addition energy for all wave-vectors accept the band-edges, $2 \pi / 3$ and $4 \pi / 3$.

There are also important effects of $O\left(U^{2} / t\right)$ which arise from the interactions between bulk and edge states. We can consider integrating out the bulk states to obtain a low energy effective action for the edge states. Due to the gapless nature of the bulk Dirac spectrum, this produces long range retarded interactions among the edge excitations. Decay processes of edge into bulk electrons are forbidden by energy-momentum conservation but the Feynman diagrams of Fig. 5 induce quartic interaction terms. For large $W$ and low energies we may calculate these interactions keeping only the low energy bulk states near the Dirac points, using the corresponding Dirac propagators. Note that we ignore interaction effects in the bulk, as discussed above. This is rather similar to an RKKY interaction. The interaction 
involving the dynamical spin operators $\left[20, \vec{S}_{U / L}(\omega, q)\right.$ on the upper and lower edge, respectively is:

$$
S_{\text {inter }}=\int \frac{d q d \omega}{(2 \pi)^{2}} \vec{S}_{U}(\omega, q) \cdot \vec{S}_{L}(-\omega,-q) J_{\text {inter }}(\omega, q, W)
$$

where

$$
J_{\text {inter }}(\omega, q, W)=2 U^{2} \int \frac{d \omega^{\prime} d k}{(2 \pi)^{2}} G\left(\omega^{\prime}, k, 0, W\right) G\left(\omega-\omega^{\prime}, q-k, 0, W\right) .
$$

Here $G(\omega, k, 0, W)$ is the bulk free electron Green's function with momentum $k$ in the $x$-direction at $y=0$ and $y=W$ with appropriate zigzag or bearded boundary conditions and projected onto the sublattices corresponding to the upper and lower edge ( $A-A$ for zigzag-bearded or $A-B$ for zigzag-zigzag). Using the linearized, Dirac dispersion relation, which is valid at small $1 / W, \omega / t$ and $q$,

$$
G_{Z B}\left(\omega, k_{x}, y=0, y^{\prime}=W\right)=\frac{2 i v_{F}^{2}}{W} \sum_{n} \frac{(-1)^{n} k_{n}^{2} \omega}{\epsilon^{2}\left(k_{x}, k_{n}\right)\left[\omega^{2}+\epsilon^{2}\left(k_{x}, k_{n}\right)\right]}
$$

where $\epsilon(\vec{k})=v_{F}|\vec{k}|$ is the Dirac dispersion relation. $G_{Z Z}$ is given by the same expression with $\omega$ replaced by $i \epsilon\left(k_{x}, k_{n}\right)$ in the numerator inside the sum. The sum over $n$ can be taken up to an arbitrary ultra-violet cut-off whose value doesn't affect the behavior at small $1 / W, \omega / t$ and $q . k_{n}=\pi n / W$ for the ZB case. Although the wave-vectors of edge modes are phase-shifted from these values in the ZZ case, this can be ignored at leading order in $1 / W$, allowing us to again use $k_{n}=\pi n / W$.

It is straightforward to evaluate $J_{\text {inter }}(\omega, q, W)$ numerically with the two types of edges. The characteristic scales for the $\omega$ and $q$ dependence of $J_{\text {inter }}$ are set by $t / W$ and $1 / W$, respectively. Since the energy scale of the interedge interaction in Eq. 21) is $U^{2} /\left(t W^{2}\right)$, it should be permissible to ignore the retardation, and evaluate $S_{\text {inter }}$ at $\omega=q=0$ to calculate the properties of low energy states. This gives:

$$
J_{Z B / Z Z}(W)=\mp c \frac{U^{2}}{t} \frac{1}{W^{2}}
$$

where the positive constant $c$ is given by the convergent sums and integral:

$$
c \equiv \frac{\sqrt{3}}{\pi} \times \sum_{n, m=1}^{\infty}(-1)^{n+m} \int_{-\infty}^{\infty} d \kappa \frac{n^{2} m^{2}}{\left(\kappa^{2}+m^{2}\right)\left(\kappa^{2}+n^{2}\right)} \frac{1}{\sqrt{\kappa^{2}+m^{2}}+\sqrt{\kappa^{2}+n^{2}}} \approx 0.20
$$

(A similar result was obtained in 4 for the $\mathrm{ZZ}$ case.) We see that the ground state for the zigzag-bearded ribbon has spin $L / 2$ while that for the zigzag-zigzag case has spin 0 , as shown above rigorously using Lieb's theorem. The remarkable fact that the change in sign of this tiny coupling drastically changes the spin of the ground state provides evidence for the polarized nature of the edge spins. There is also a large manifold of low energy states, which are simply the eigenstates of $J_{Z Z} \vec{S}_{T} \cdot \vec{S}_{B}$ with $S_{T}=S_{B}=L / 6$ (in the ZZ case).

Another important effect of $O\left(U^{2} / t\right)$ is the intra-edge interaction, independent of $W$. For a zigzag edge by integrating out the low-energy bulk excitations, the spin part is:

$$
S_{\text {intra }}=\int \frac{d q d \omega}{(2 \pi)^{2}} \vec{S}(\omega, q) \cdot \vec{S}(\omega, q) J_{\text {intra }}(\omega, q)
$$

with

$$
J_{\text {intra }}(\omega, q)=2 U^{2} \int_{k, \omega^{\prime} / v_{f}<\Lambda} \frac{d \omega^{\prime} d k}{(2 \pi)^{2}} G\left(\omega^{\prime}, k, y=y^{\prime}=0\right) G\left(\omega-\omega^{\prime}, q-k, y=y^{\prime}=0\right)
$$

Now the free bulk Green's function, with zigzag edge boundary conditions, may be evaluated for a semi-infinite system, giving, at small $k_{x}$ (measured from a Dirac point) and small $\omega$ :

$$
G\left(\omega, k_{x}, y=y^{\prime}=0\right) \approx 2 i v_{F}^{2} \int \frac{d k_{y}}{2 \pi} \frac{k_{y}^{2}}{\left(v_{F} k\right)^{2}} \frac{\omega}{\omega^{2}+\left(v_{F} k\right)^{2}}
$$

By using this green function, the $J_{\text {intra }}$ of Eq. 25) is ultraviolet divergence and the integral should be cut off at some point $\Lambda$. Although the resulting $J_{\text {intra }}(\omega, q)$ is cut off dependent, by ignoring the weak retardation, the corrections to 

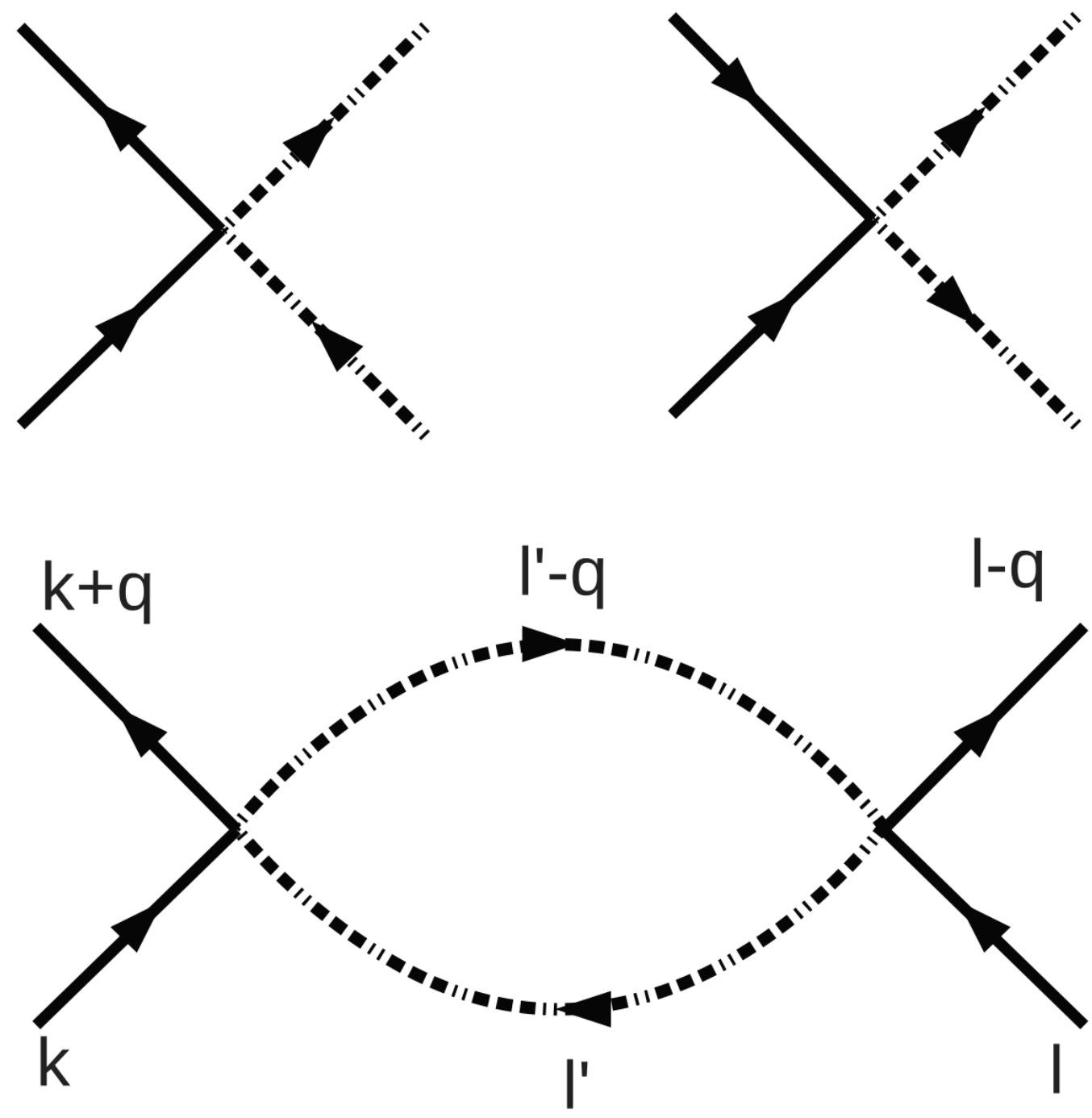

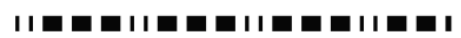

Bulk propagator

Edge propagator

FIG. 5. Feynman diagrams inducing edge interactions from integrating out bulk states. 
the energy of the excitons is proportional to $J_{\text {intra }}(0, q)-J_{\text {intra }}(0,0)$, which is cut off independent. For small $\omega$ and $q$ these $O\left(U^{2}\right)$ intra-edge interactions become more singular than the $O(U)$ terms by logarithmic factors of $q^{2} \ln q$, giving for example the final equation in the main paper.

As mentioned above, Eq. (25) only includes the effect of low energy bulk excitations; it is still possible that the high energy bulk excitations could wipe out this singularity of the exciton dispersion relation. By using the exact form of the bulk wavefunctions one can determine the exact intra-edge interaction of $O\left(U^{2}\right)$. This has a more complicated form than Eq. (25). Nonetheless, it can be shown that the only part of this interaction which contributes to this $\ln q$ singular correction to the excition dispersion relation is the contribution from low energy bulk excitations of the form of Eq. 25.

We leave a more detailed study of these effects of $O\left(U^{2}\right)$ and higher for the future. A reasonable approach might be to ignore the bulk interactions, since they are irrelevant, but analyse the bulk-edge Hubbard interactions using the renormalization group. This corresponds to a novel type of boundary critical phenomena in which the bulk is a massless $(2+1)$ dimensional Dirac liquid and the edge is a one-dimensional spin-polarized semi-metal. The arguments based on Lieb's theorem imply that the edge magnetic moment remains stable against weak interactions.

We would like to thank Ion Garate for his collaboration in the early stages of this project and Pawel Hawryak for interesting discussions. The research was supported in part by NSERC and CIfAR. We also thank the Galileo Galilei Institute where this work was completed.

[1] A. H. Castro Neto, F. Guinea, N. M. R. Peres, K. S. Novoselov and A. K. Geim, Rev. Mod. Phys. 81, 109 (2009).

[2] M. Fujita, K. Wakabayashi, K. Nakada, K. Kusakabe, J. Phys. Soc. Jpn. 65, 1920 (1996).

[3] C. Tao et al. Nat. Phys. 7, 616 (2011).

[4] J. Jung, T. Pereg-Barnea, A. H. MacDonald, Phys. Rev. Lett. 102, 227205 (2009).

[5] J. Jung, A. H. MacDonald, Phys. Rev. B 79, 235433 (2009).

[6] Y.-W. Son, M. L. Cohen, and S. G. Louie, Phys. Rev. Lett. 97, 216803 (2006).

[7] Y.-W. Son, M. L. Cohen, and S. G. Louie, Nature 444, 347 (2006).

[8] T. Hikihara, X. Hu, H.-H. Lin, and C.-Y. Mou Phys. Rev. B 68, 035432 (2003).

[9] S. Dutta, S. Lakshmi, and S. K. Pati, Phys. Rev. B 77, 073412 (2008).

[10] H. Feldner, Z.Y. Meng, A.Honecker, D.Cabra, S. Wessel, and F.F. Assaad, Phys. Rev. B 81, 115416 (2010).

[11] W. Y. Kim and K. S. Kim, Nature Nanotechnology 3, 408 (2008).

[12] J. Kunstmann, C. Ozdogan, A. Quandt, H. Fehske, Phys. Rev. B 83, 045414 (2011).

[13] M. J. Schmidt and D. Loss, Phys. Rev. B 82, 085422 (2010).

[14] D. J. Luitz, F. F. Assaad, M. J. Schmidt, Phys. Rev. B 83, 195432 (2011).

[15] E..H. Lieb, Phys. Rev. Lett. 62, 1201 (1989).

[16] In the case where $L$ is a multiple of 3 there are states exactly at the band edges $\pm 2 \pi / 3$. We choose not to include them in the edge Hamiltonian since they are delocalized over the entire ribbon.

[17] For the case of $t_{2}$ this is shown in K. Sasaki, S. Murukami and R. Saito, Appl. Phys. Lett. 88, 113110 (2006). For the case of the edge potential, it is shown in 13 .

[18] Z. Y. Meng, T. C. Lang, S. Wessel, F. F. Assaad, A. Muramatsu, Nature 464, 847 (2010).

[19] Closely related observations were made in M.J. Schmidt, Phys. Rev. B 84, 241403(R) (2011).

[20] There are additional inter-edge interactions involving the number operators and pair creation operators on upper and lower edges which will be discussed in a later article. However these have little effect at large $W$. The spin term dominates because the edges are spin polarized. 